
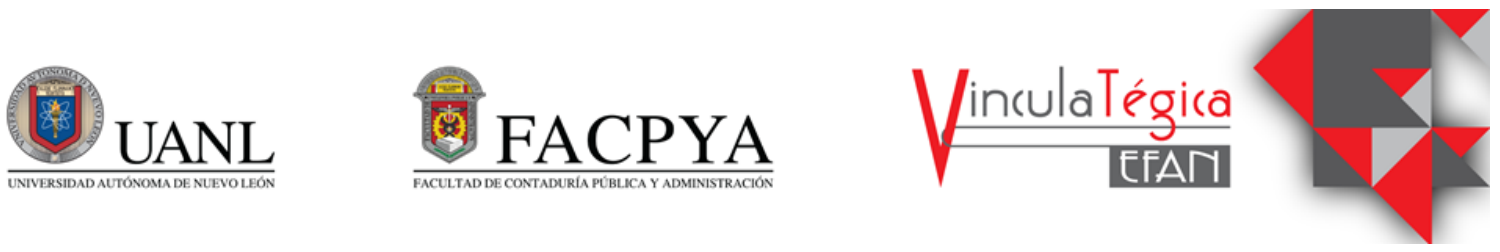

\title{
Los Sistemas de Información Tecnológica como herramienta para la competitividad empresarial en las MiPyMES
}

\author{
Benn Katsamudanga Tonderai ${ }^{1}$, Abel Partida Puente ${ }^{2}$ y María Margarita Carrera Sánchez ${ }^{3}$ \\ 1,2,3 Universidad Autónoma de Nuevo León, Facultad de Contaduría Pública y Administración, \\ tonderaibenn@yahoo.com, abelpartida@hotmail.com, magaly_carrera@hotmail.com, Av.Universidad s/n \\ Cd. Universidad C.P. 66455. San Nicolás de los Garza, Nuevo León, México.
}

Información del artículo revisado por pares

Fecha de aceptación: junio-2021

Fecha de publicación en línea: diciembre-2021

DOI: https://doi.org/10.29105/vtga7.1-156

\begin{abstract}
There are few IT management programs in the veterinary sector, which only a minority know how to use in their daily work. Without the use of information technology systems, business operations, performance and competitiveness would be affected. The objective of this research is to determine if the use of technological information systems among SMEs of the veterinary services sector in Mexico, influences business competitiveness and in the end proposing a theoretical model that supports the objective of this research.
\end{abstract}

\section{Keywords}

Business Competitiveness, Veterinary Management, Use of Information Technology Systems.

JEL Code: M1, M13, M15, Y10

\section{Resumen}

Existen pocos programas de gestión de informática en el sector veterinario, que solo una minoría saben usar en su trabajo diario. Sin la utilización de los sistemas de información tecnológica, el funcionamiento, el desempeño y la competitividad empresarial se verían afectados. El objetivo de la presente investigación es determinar si el uso de los sistemas de información tecnológica en el sector de las MIPYMES de servicios veterinarios en México, tienen un efecto en la competitividad empresarial, proponiendo un modelo teórico que sustenten el objetivo de la presente investigación.

\section{Palabras claves}

Competitividad Empresarial, Gestión Veterinaria, Uso de Sistemas de Información Tecnológica

Código JEL: M1, M13, M15, Y10 


\section{Introducción}

Ha sido determinado que existen pocos programas de gestión veterinaria, que solo una minoría saben usar en su trabajo diario. Ejemplos de los programas que han dominado el mercado de la gestión de los servicios veterinarios son GVET, SAMI, QVET, QSOFT y Panther. Algunos de sus características que son importante para la gestión de las MIPYMES de los servicios veterinarios incluyen la captura de las historias clínicas y tratamientos; gestión financiera y manejo de información personal de los propietarios y comunicación con ellos también. Además, existe la ventaja de gestionar los recursos de estas entidades. Sin la utilización de los sistemas de información tecnológica habrá mayores deficiencias que afectaría, de manera negativa, como la función de las MIPYMES de este sector y también el desempeño empresarial, que al final puede resultar en la disminución de la competitividad empresarial.

Para desarrollar el marco teórico se va a considerar las próximas teorías principales sobre el uso de los sistemas de información tecnológica y la competitividad empresarial (tabla 1).

Tabla 1: Teorías y modelos relacionados con el uso de sistemas de información tecnológica y la competitividad empresarial

\begin{tabular}{ll}
\hline Autores & Teoría y modelos \\
\hline Abdullah et al., (2012)., Abu, (2015) & $\begin{array}{l}\text { Technology Acceptance Model, Theory Reasoned Action, Unified } \\
\text { Theory of Acceptance and Use of Technology, Theory of Planned } \\
\text { Behavior }\end{array}$ \\
\hline Abdulhakeem, (2017) & $\begin{array}{l}\text { Resource based Theory, Technology Organization Environment } \\
\text { Chairoel y Riski, (2018)., Ibrahim, } \\
\text { (2018). }\end{array}$ \\
$\begin{array}{l}\text { Framework, Perceived E-Readiness Model, Modelo Motivacional, } \\
\text { Modelo de utilización de computadores personal, teoría Diffusion of } \\
\text { Innovation, teoría de cognitiva social, }\end{array}$ \\
\hline Bi, Davison, Smyrnios, (2016). & Resource Based View \\
\hline \multicolumn{1}{c}{ Fuente: Elaboración propia } &
\end{tabular}

Existen varios modelos que han sido utilizados para explicar el por qué algunos MIPYMES utilizan sistemas de información tecnológica para aumentar sus niveles de competitividad empresarial. Los modelos incluyen el modelo TAM, (Technology Acceptance Model), que fue desarrollado utilizando la teoría TRA, (Theory Reasoned Action). Postula que el uso de los sistemas de la información tecnológica depende de las percepciones de la utilidad y la facilidad de ellos para las organizaciones. Si una MIPYME, del sector de los servicios veterinarios, se considera que una forma de los sistemas de información tecnológica le apoyaría en aumentar sus niveles de competitividad empresarial, existiría mayores chances que lo usará (Abdullah et al., 2012., Abu, 2015)

La modificación de TAM produjo la teoría de UTAUT, (Unified Theory of Acceptance and Use of Technology) que postulaba que las funciones esperadas, esfuerzos necesitados para su uso, influencias sociales, son determinantes directos al uso de los sistemas de información tecnológicas entre las MIPYMES para aumentar sus niveles de competitividad. Habrá mayores chances de adopción y uso de los sistemas de información tecnológicas si se ofrecen más funciones que sus competidores no tienen. Además, el sexo, edad, experiencia y la voluntad al usarlos, significando que las características organizacionales pueden ser un factor para considerar para 
aumentar la competitividad empresarial. También la teoría TPB, (Theory of Planned Behavior), determinó que el uso de los sistemas de información tecnológica está determinado por las intenciones de los individuos, para los que les interesa a aumentar la competitividad empresarial. En todos estos casos estas teorías deberían ser puestos en contexto de las características de los administradores de las MIPYMES, organizaciones y los ambientes externos, como ya son factores que afectan la decisión al usarlos (Abdullah et al., 2012., Abu, 2015)

No obstante, Abdulhakeem, (2017) explicó que las teorías antes mencionadas, incluyendo la teoría RBT, (Resource Based Theory) para países en desarrollo, no pueden explicar individual y completamente las razones porque algunas MIPYMES deciden usar sistemas de información tecnológica. Razones siendo que existen diferencias en la competitividad empresarial entre países desarrollado y no desarrollado. Se propusieron el uso integrado de la teoría TOE, (Technology Organization Environment Framework) y la teoría de PERM, (Perceived E-Readiness Model). Ambas destacan, porque se enfatizan conjuntamente que la tecnología, organizaciones, el ambiente son factores importantes para relacionar el uso de los sistemas de información tecnológica y la competitividad empresarial. Otros modelos y teorías importante y que son complementarias a los mencionados son; Modelo Motivacional (MM), teoría combinada de TAM y TPB, modelo de utilización de computadores personal (MPCU), teoría Diffusion of Innovation (DOI) y la teoría de cognitiva social (SCT) (Chairoel y Riski, 2018., Ibrahim, 2018).

La teoría RBV, (Resource Based View), ha sido utilizado para explicar por qué las MIPYMES pueden tener ventajas competitivas si se utilizan los recursos e información que sus competidores no tienen (Bi, Davison, Smyrnios, 2016). Se determinaron en un estudio de las MIPYMES en Córdoba, Argentina que el uso de sistemas de información tecnológica relacionaba de forma positiva con a un aumento de competitividad empresarial y esto fue mostrado por; una reducción de costos de transacciones, mejor comunicación con los clientes y proveedores, integración de información y toma de decisiones empresariales, mejora la atención a los clientes y por ende la competitividad empresarial (Jones et al., 2016).

\section{Marco Teórico}

2.1. Definición conceptual: Uso de los Sistemas de Información Tecnológica

El uso de los sistemas de información tecnológica incluye la utilización del internet, así como su infraestructura, incluidos los equipos y programas informáticos, y aquellas tecnologías que procesan o transmiten información para mejorar la eficacia de las personas y organizaciones (Ghobakhloo, 2012). Existe la captura, almacenamiento, procesamiento y distribución de la información para apoyar la toma de decisiones, el control, análisis y visión en una organización (Castañeda Parra, 2013). Riascos Erazo, (2016) lo considera como uso del hardware, software y sistemas de telecomunicaciones.

Es el uso de formatos que pueden ser las voces, imágenes, acústica o electromagnética para organizar o transmitir información de una organización (Montoya Macario y Acosta Gonzaga, 2019). Mientras Nguyen et al., (2020) y Ibrahim, (2018) agregaron que el uso de los sistemas de información 
tecnológica es la utilización de herramientas electrónica de información, nubes de información, aplicaciones de posicionamiento geográfica y sistemas de inteligencia artificial.

Para aclarecer la implementación del uso de sistemas de información tecnológica, fue determinado en una industria de bordado de uniformes en Querétaro que las siguientes etapas deberían ser seguido al adoptar y usar sistemas de información tecnológica en las MIPYMES;

1. Comunicación con una línea fija o un teléfono móvil, ya que esto permite una comunicación con sus proveedores y clientes.
2. Adaptar una computadora personal con software básico, incluso sin conectividad a Internet.

3. La adopción de Internet, con el correo electrónico, el intercambio de archivos, la creación de sitios web y el comercio electrónico.

4. Adopción de las herramientas más complejas como software de gestión de inventario, contabilidad o expedientes clínicos. (Montoya Macario y Acosta Gonzaga, 2019).

La siguiente tabla 2 muestra los autores que han contribuido a la definición conceptual sobre el uso de sistemas de información tecnológica que se va a ser utilizado en esta investigación.

Tabla 2: Definiciones sobre el uso de sistemas de información tecnológica

\begin{tabular}{|c|c|c|}
\hline Autor & Definición & Dimensiones \\
\hline Ghobakhloo, (2012). & $\begin{array}{l}\text { La utilización del internet, así como su } \\
\text { infraestructura, incluidos los equipos y } \\
\text { programas informáticos, y aquellas } \\
\text { tecnologías que procesan o transmiten } \\
\text { información para mejorar la eficacia de las } \\
\text { personas y organizaciones }\end{array}$ & $\begin{array}{l}\text { Formas y eficiencia } \\
\text { organizacional }\end{array}$ \\
\hline Castañeda Parra, (2013). & $\begin{array}{l}\text { La captura, almacenamiento, } \\
\text { procesamiento y distribución de la } \\
\text { información para apoyar la toma de } \\
\text { decisiones, el control, análisis y visión en } \\
\text { una organización. }\end{array}$ & Proceso de uso \\
\hline Riascos Erazo, (2016) & $\begin{array}{l}\text { Es el uso del hardware, software y sistemas } \\
\text { de telecomunicaciones }\end{array}$ & Tipos \\
\hline $\begin{array}{l}\text { Nguyen et al., (2020) e Ibrahim, } \\
\text { (2018) }\end{array}$ & $\begin{array}{l}\text { Es la utilización de herramientas } \\
\text { electrónica de información, nubes de } \\
\text { información, aplicaciones } \\
\text { posicionamiento geográfica y sistemas de } \\
\text { inteligencia artificial. }\end{array}$ & Formas y uso \\
\hline $\begin{array}{l}\text { Montoya Macario y Acosta } \\
\text { Gonzaga, }(2019)\end{array}$ & $\begin{array}{l}\text { Es el uso de formatos que pueden ser las } \\
\text { voces, imágenes, acústica o } \\
\text { electromagnética para organizar o } \\
\text { transmitir información de una organización }\end{array}$ & Formas y uso \\
\hline
\end{tabular}

Fuente: Elaboración propia

Se consideraron todas las muestran la existencia de los medios de definiciones antes mencionado porque utilización, procesos de utilización, 
equipos físicos y no físicos y las ventajas de utilización. Además son relevante y pueden ser identificado en el sector de los servicios veterinarios.

Para el análisis sobre el uso de sistemas de información tecnológica las siguientes preguntas pueden ser usado (Ghobakhloo, 2012., Castañeda Parra, 2013., Riascos Erazo, 2016., Pérez Rendón et al., 2017., Ibrahim, 2018., Sánchez Limón y De la Garza Cárdenas, 2018., Montoya Macario y Acosta Gonzaga, 2019., Nguyen et al., 2020).

¿Consideras el uso de internet importante para almacenar información de tu empresa?

¿Consideras el uso de internet importante para procesar información de tu empresa?

¿Consideras el uso de internet importante para distribuir información de tu empresa?

¿Consideras el uso de internet importante para la toma de decisiones de tu empresa?

¿El uso de sistemas de información tecnológica apoya el control de una organización?

¿El uso de sistemas de información tecnológica apoya el análisis en una organización?

¿El uso de sistemas de información tecnológica apoya la visión en una organización?

¿Consideras el uso de internet importante para capturar la información de tu empresa?

¿Los equipos (hardware) son incluidos en el uso de información tecnológica?
¿Los programas informáticos (software) son incluidos en el uso de información tecnológica?

¿Las tecnologías que procesan o transmiten información (sistemas de telecomunicaciones, herramientas electrónicas de información, nubes de información, aplicaciones de posicionamiento geográfica y sistemas de inteligencia artificial) son incluidos en el uso de información tecnológica?

\subsection{Competitividad Empresarial en las MIPYMES}

No obstante, la competitividad empresarial ha sido determinada como la habilidad de las empresas a diseñar, producir y poner productos y servicios superiores en valor y apreciación de los consumidores en comparación con los de sus competidores. En la mayoría de los casos esto se ve reflejado en su desempeño y los niveles de las ganancias y las cuotas en el mercado (Ambastha \& Momaya, 2004). Además, los siguientes indicadores sobre la competitividad empresarial deberían ser analizados al comparar empresas del mismo sector; costo, precio, volumen de producción y servicios, niveles de calidad, presencia en el mercado, actividades de innovación, flexibilidad y adaptación a los cambios del entorno, así como la evaluación periódica de los esfuerzos de la empresa por mejorar y tales indicadores (Enríquez., Adame \& Camacho, 2016).

Hasta el presente la competitividad empresarial ha sido determinado a nivel nacional, estatal y municipal pero nunca han sido determinado a nivel empresarial, pero Sariannidis, (2019) propusó la siguiente ecuación que puede ser aplicado a las MIPYMES del sector de servicios veterinarios.

\section{Índice de competitividad empresarial = CMS + CROA}

Donde, CMS (Change in market share between two years during the period under investigation) es el cambio en la cuota del 
mercado entre dos años, durante el periodo bajo investigación. CROA (Change in return of assets between two years for the period under investigation) cambio en el valor de propiedades durante dos años seguidos.

\subsection{Relaciones entre uso de sistemas de información tecnológica y la competitividad empresarial}

Según el INEGI en el año 2017, existían 71,340,853 personas que usaban el internet en México, siendo el 96,9 \% empleándolo para obtener información; y el $16,6 \%$ para ordenar o comprar productos. Además, el $96 \%$ utilizaban la computadora para usos empresariales mientras el $93.6 \%$ de estas empresas admitieron usar Internet para el negocio. El $58 \%$ de las MIPYMES mexicanas anunciaba su negocio en el internet mientras el $22 \%$ lo utilizaba para dar servicio a sus clientes y sólo un $27 \%$ para hacer compras o para pedidos a sus proveedores. Sólo un $39 \%$ de dichas empresas tenían su propia página de Internet. (Leyva Morales et al., 2017., Lizama Juárez et al., 2020).

Como ya se han determinado que para mantenerse viable en el mundo que esta tan globalizado, las MIPYMES deberían implementar estrategias que apoya el aumento de los niveles de competitividad empresarial. En México el gobierno fue obligado a introducir el Plan Nacional Digital que abogó por el uso y desarrollo de los sistemas de información tecnológica (Montoya Macario y Acosta Gonzaga, 2019). Desde entonces la mayoría de las MIPYMES consideran los sistemas de información tecnológica como un catalizador a los procesos de negocios y que afecta positivamente la competitividad empresarial (Adane, 2018).

Fue reportado que, en países como Estados Unidos, España y Portugal, que había sólo el $13 \%, 10 \%$ y $12 \%$, respectivamente, de los centros veterinarios que usaban sistemas de información tecnológica en sus gestiones diaria (Troncoso., López., Fischer., Luzio., Muñoz y Carrasco, 2013). Considerando estos bajos resultados implicaría una necesidad de investigar sobre el uso de los sistemas de información tecnológica entre las MIPYMES del sector de servicios veterinarios en el municipio de Monterrey, Nuevo León, México, $(\mathrm{p}<0.05, \beta=1.2)$, cuyos resultados pueden estar similar a los antes mencionados (Leyva Morales et al., 2017., Lizama Juárez et al., 2020).

Chege y Wang, (2019) en su revisión de la literatura sobre el impacto que tiene el uso de sistemas de información tecnológica sobre la creación de empleo, se agregaron que existe una relación positiva y de la misma manera un aumento de trabajadores satisfecho con su ambiente estará apuntado hacia el aumento en los ingresos de las MIPYMES, un reflejo de mayor competitividad empresarial. Mientras Medina Torres, (2013), después de aplicar un instrumento de medición sobre uso de las computadoras y el internet en Celaya, Guanajuato, mencionó que la decisión a usar estos tipos de sistemas de información tecnológica pudiera estar relacionado con la satisfacción de los clientes.

Además de lo antes mencionado, la utilización de los sistemas de información tecnológica ofrece varias ventajas competitivas que incluyen indirectamente una mayor cuota en el mercado. Fue evidenciado en un estudio realizado con los MIPYMES, de los servicios, durante el periodo de la pandemia de COVID 19 en Bogotá Colombia que existe una relación positiva entre uso de sistemas de tecnología y una mayor ocupación en el mercado (Rodríguez Melo, 2012., Ochoa Guevara et al., 2020). También fue determinado en un estudio de digital marketing de las MIPYMES en México que el 39,8 \% usaban Facebook, 19,9 \% WhatsApp mientras aproximadamente el $13 \%$ usaban Instagram para la promoción y la venta de los productos y los servicios (Lizama Juárez, 2020). Ya, están considerados como sitios idóneos para la publicidad de los productos y servicios que ofrecen las MIPYMES (Ixmatlahua, 2015).

No obstante, en un estudio de las MIPYMES involucrado en el sector de seguridad privada en Kenia se demostraron que los costos para adquirir el hardware, 
software y el entrenamiento de los trabajadores fueron las causas principales de la no utilización de sistemas de información tecnológica y bajos niveles del desempeño (Maghanga, 2017). En otro sentido ha sido determinado que existe una resistencia al uso de sistemas de información tecnológica por las MIPYMES y algunos de las razones para las MIPYMES en Colombia, siendo la falta de necesidad de usarlos, trabajadores mal capacitados para adoptar su uso y los altos costos para adquirirlos (Rodríguez Melo, 2012). Aunque las MIPYMES de Santiago de Cali en 2017 mostraron que el uso de los sistemas de información tecnológica brindaría nuevas oportunidades $(\alpha=0.556)$, también tuvieron percepciones negativas sobre los costos de adquirirlos. (Aguilera Castro., Ávila Fajardo., Solano Rodríguez, 2017). En todas estas situaciones la competitividad empresarial estuvo en niveles negativos, $(p>$ 0.062, $\beta=0.64$ ), (Jones et al 2016., Montoya Macario y Acosta Gonzaga, 2019).

Se determinaron, utilizando la información de un estudio exploratorio de las MIPYMES en Malasia, que existen barreras para el uso de sistemas de información tecnológica como la seguridad percibida, privacidad, niveles de educación de los empleadores, costos de adquisición y la falta de regulaciones. Del mismo modo, era probable que algunos elementos influyan significativamente, como la calificación académica del CEO, la pasión, el compromiso, el conocimiento tecnológico, estilo de liderazgo, conciencia tecnológica y su voluntad de aprender, hábiles y competentes personales, cultura organizacional y orientación a la cliente basada en las tendencias del mercado y el gobierno (Ikumoro, 2019).

Utilizando información obtenido de un estudio descriptivo sobre uso de comercio electrónica, con 167 encuestados, fue observado que el $80 \%$ de las MIPYMES no tenían uso de comercio electrónica y páginas de web de sus empresas (Sin., Osman., Salahuddin., Abdullah., Lim y Sim, 2016., Cano y Rojas, 2017). Estos resultados mostraron el grado de uso de sistemas de información tecnológica entre las MIPYMES en Malasia. También, se notaron en un estudio sobre las brechas tecnológicas en México, utilizando regresiones ergonométricas con datos de INEGI, que el uso de los sistemas de información tecnológica está afectado por la edad, el nivel de escolaridad, el ingreso, la ocupación, las habilidades digitales y la ubicación geográfica de los propietarios de las microempresas (Domínguez, 2018). Finalmente, como fue determinado a partir de un estudio descriptivo donde se participaron 270 gerentes de las MIPYMES de Cali, Colombia, se concluyeron que la disponibilidad de la información no financiera como uso de sistemas de información tecnológica con lleva a la buena toma de decisiones y esto proporciona pasos hacia ventajas competitivas (González González, 2010).

\section{Metodología}

Se muestra los resultados de la búsqueda en la literatura que aporta información al marco teórico de la investigación: Los Sistemas de Información Tecnológica como herramienta para la competitividad empresarial en las MIPYMES. Los bases de datos que fueron utilizados incluyen EBSCO, JCR, SCOPUS y Google Académico. Los artículos usados, fueron obtenidos usando las palabras claves con una antigüedad de 5 años. Principalmente, se hace un recorrido de las teorías, las definiciones y relaciones sobre el uso de los sistemas de información tecnológica con la competitividad empresarial. Finalmente se establece la pregunta, objetivo, hipótesis y la conclusión de la investigación.

\section{Pregunta de Investigación}

¿El uso de los sistemas de información tecnológica genera un efecto positivo en la competitividad empresarial de las MIPYMES del sector de servicios veterinarios en Monterrey, Nuevo León, México?

\section{Objetivo}

Determinar a través de un modelo teórico que el uso de los sistemas de información tecnológica tiene un efecto sobre la 
competitividad empresarial de las MIPYMES del sector de servicios veterinarios en Monterrey, Nuevo León, México.

\section{Conclusión}

Considerando el objetivo y la pregunta de la investigación anunciado como: ¿El uso de los sistemas de información tecnológica generan un efecto en la competitividad empresarial de las MIPYMES del sector de servicios veterinarios en Monterrey, Nuevo León, México?, se concluye que el uso de los sistemas de información tecnológica genera un efecto positivo en la competitividad empresarial de las MIPYMES del sector de servicios veterinarios en Monterrey, Nuevo León, México. Además, se propone un modelo teórico, figura 1 , que muestra una relación entre el uso de los sistemas de información tecnológica y la competitividad empresarial.

Figura 1: Modelo teórico sobre la relación entre uso de sistemas de información tecnológica y la competitividad empresarial.

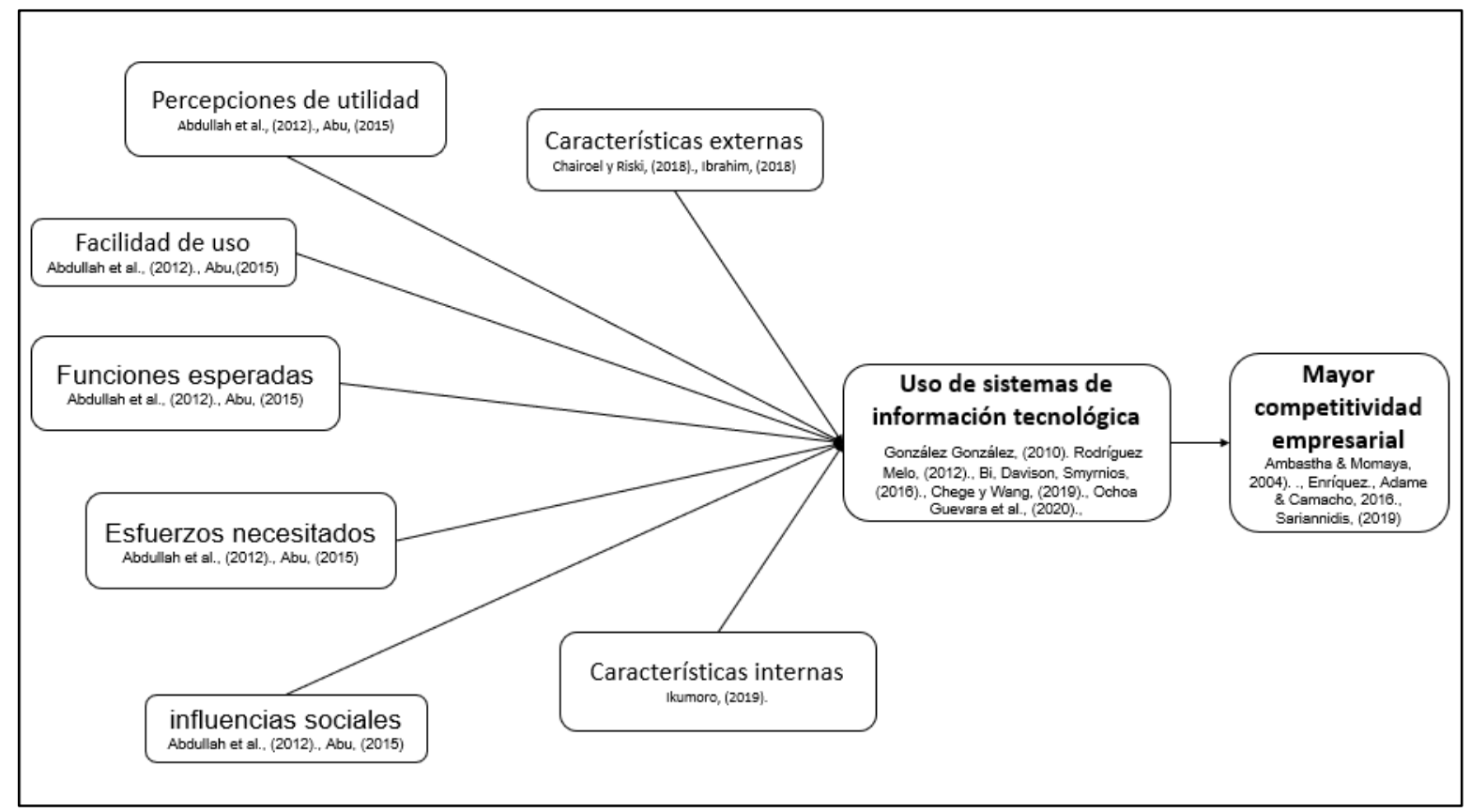

Fuente: Elaboración propia 


\section{Referencias}

Abdulhakeem, I., Edwards, H and McDonald, S. (2017). Ecommerce adoption in Developing Countries SMEs: What Do the Prevailing Theoretical Models Offer Us? In: 4th International Conference on Commerce, 18-20 Sep 2017, Putrajaya, Malaysia. En: http://sure.sunderland.ac.uk/id/eprint/8301/

Abdullah, N. H., Shamsuddin, A., Wahab, E and Hamid, N. A. (2012). Preliminary Qualitative Findings on Technology Adoption of Malaysian SMEs. 2012 IEEE Colloquium on Humanities, Science \& Engineering Research (CHUSER 2012), December 3-4, 2012, Kota Kinabalu, Sabah, Malaysia. Faculty of Technology Management and Business Universiti Tun Hussein Onn Malaysia, Batu Pahat, Johor, Malaysia

Abu, F., Jabar, J., Yunus, A. R. (2015). Modified of UTAUT Theory in Adoption of Technology for Malaysia Small Medium Enterprises (SMEs) in Food Industry. Aust. J. Basic \& Appl. Sci., 9(4) , 2015: pp 104-109

Adane, M. (2018). Cloud computing adoption: Strategies for Sub-Saharan Africa SMEs for enhancing competitiveness. African Journal of Science, Technology, Innovation and Development, 10:2, pp 197-207, DOI: 10.1080/20421338.2018.1439288

Aguilera-Castro, A., Ávila-Fajardo, GP., Solano-Rodríguez, OJ. (2017). Las TIC en la formulación estratégica de las pymes de Santiago de Cali - Colombia. Entramado. Vol. 13 No. 1, 2017 (enero - junio). En: http://dx.doi.org/10.18041/entramado.2017v13n1.25106

Ambastha, A \& Momaya, K. (2004). Challenges for Indian software firms to sustain their global competitiveness. Singapore Management Review, 26(2), pp 65-78. https://go.gale.com/ps/anonymous?id=GALE\%7CA1 19370567\&sid=googleScholar\&v=2.1\& $\mathrm{it}=\mathrm{r} \&$ linkaccess $=$ abs\&issn $=01295977 \& \mathrm{p}=\mathrm{AONE} \& \mathrm{sw}=\mathrm{w}$

Bi, R., Davison, R.M., Smyrnios, K.X. (2016). E-business and fast growth SMEs. Small Bus Econ. DOI 10.1007/s11187-016-9788-8

Castañeda Parra, L.I. (2013). Los sistemas de información contable una herramienta para la gestión integral de las Pymes. Revista de la Agrupación Joven Iberoamericana de Contabilidad y Administración de Empresas (AJOICA). № 11 - 2013. pp. 31 - 40.

Chairoel, Riski, TR. (2018). Internal and external factor influence ICT adoption: A case of Indonesian SMEs. JMK, VOL. 20, NO. 1, MARCH 2018, 38-44 DOI: 10.9744/jmk.20.1.38-44. ISSN 1411-1438 print / ISSN 2338-8234 online

Chege, SM \& Wang, D. (2019). Information technology innovation and its impact on job creation by SMEs in developing countries: an analysis of the literature review. Technology Analysis \& Strategic Management. https://doi.org/10.1080/09537325.2019.1651263

Enríquez, L.A., Adame, M.G \& Camacho, R.R. (2016). Factores que impactan la competitividad de las pymes. Mercados y $\quad$ Negocios, $116-136$. http://mercadosynegocios.cucea.udg.mx/index.php/MYN/article/view/5149

Ghobakhloo, M., Hong, T.S., Sabouri, M.S y Zulkifli, N. (2012). Strategies for Successful Information Technology Adoption in Small and Medium-sized Enterprises. Information 2012, 3, pp 36-67; doi:10.3390/info3010036

González González, P. (2010). Fuentes de información, indicadores y herramientas más usadas por gerentes de MIPYME en Cali, Colombia. Contaduría y Administración, No. 232, septiembrediciembre 2010: pp 83-108.

Ibrahim, A.M. (2018). Factors Influencing Acceptance and Use of ICT Innovations by Agribusinesses. Journal of Global Information Management Volume $26 \bullet$ Issue 4 • OctoberDecember 2018

Ibrahim, A.M., Hassan, M.S., Gusau, A.L. (2018). Factors Influencing Acceptance and Use of ICT Innovations by Agribusinesses. Journal of Global Information Management Volume 26. Issue 4. October-December 2018. DOI: 10.4018/JGIM.2018100107 
Ikumoro, A.O., Jawad, M.S. (2019). Intention to Use Intelligent Conversational Agents in eCommerce among Malaysian SMEs: An Integrated Conceptual Framework Based on Tritheories including Unified Theory of Acceptance, Use of Technology (UTAUT), and T-O-E. International Journal of Academic Research in Business and Social Sciences, 9(11), pp 205235. En: http://dx.doi.org/10.6007/IJARBSS/v9-i11/6544

Ixmatlahua, S.D., Raygoza, R.O., Romero, O., Uribe, F., Vargas, E.J. (2015). Metrópoli Digital: Una plataforma Web para la inclusión integral de las PyMES, Sociedad y Gobierno en el uso de las Tecnologías de la Información en la región de las Altas Montañas del estado de Veracruz, México. RISTI, $N .^{o}$ E3, 03/2015. DOI: 10.17013/risti.e3.43-54

Jones, C., Motta, J y Alderete, M.V. (2016). Gestión estratégica de tecnologías de información y comunicación y adopción del comercio electrónico en MIPYMES de Córdoba, Argentina. Estudios Gerenciales 32 (2016). pp 4-13. En: http://dx.doi.org/10.1016/j.estger.2015.12.003

Leyva Morales, C., Caro Encalada, M., Hernández Guevara, A y Vázquez Alejandro, A. (2017). Tecnologías de información y comunicación en pymes exportadoras. El caso de Mérida, Yucatán. Revista de Economía - Vol. XXXIV - Núm 89. Julio a diciembre de 2017: pp 101-123

Lizama, Y., Matos, L. y Beltrán, R. (2020). Online marketing: rentabilidad al alcance de las mipymes. Revista Visión Contable, 21, 57 - 76. En: https://doi.org/10.24142/rvc.n21a3

Maghanga, E. (2017). Challenges affecting use of i.c.t by small \& medium sized enterprises (SMEs) in KENYA: A case study of Tsavo Securities Ltd. Journal of Entrepreneurship and Project Management. Vol.2, Issue No.2, pp 1-16, 2017. En: https://doi.org/10.47941/jepm.133

Medina Torres, M.G., Villalón Guzmán, M.T., Gutiérrez Rosas, P.T. (2013). Uso del Internet como TIC en las MIPYMES de Celaya, Guanajuato. En: http://www.itcelaya.edu.mx/ojs/index.php/pistas/article/view/1255/1063

Montoya Macario, E.D y Acosta Gonzaga, E.A. (2019). Adopción de TIC en las PyMEs de la industria del bordado y confección de uniformes en el Estado de Querétaro. Revista Multidisciplinaria de Avances de Investigación ISSN: 2448-5772, vol. 5 núm. 2, mayo-agosto 2019, México

Nguyen, L.P., Pham, V.H.T. (2020). Trade of ICT Products, Government, and Economic Growth: Evidence from East Asia-Pacific Region. Journal of Asian Finance, Economics and Business Vol 7 No 8 (2020). pp 175-183. doi: 10.13106/jafeb.2020.vol7.no8.175

Ochoa Guevara, S.P., Medina Acero, C., Santamaría Burgos., Ramírez Campos, DI., Paramo Renza, EA., Ochoa Guevara, NE. (2020). Reto de las MIPYMES para incursionar en el marketing digital en tiempos de COVID-19. RHS. Revista. Humanismo. Soc. 8(2): 132 - 145, diciembre 2020 / ISSNe 2339-4196. En: https://doi.org/10.22209/rhs.v8n2a09

Peña Lapeira C.J., Pereira Bolaños C.A. (2019). ICT and Business Inclusion in the Southern Communities of the City of Bogotá - Colombia. In: Botto-Tobar M., Barba-Maggi L., González-Huerta J., Villacrés-Cevallos P., S. Gómez O., Uvidia-Fassler M. (eds) Information and Communication Technologies of Ecuador (TIC.EC). TICEC 2018. Advances in Intelligent Systems and Computing, vol 884. Springer, Cham. En: https://doi.org/10.1007/978-3-03002828-2_19

Pérez Rendón, L., Ramírez Segovia, N., Topete Gómez, E. (2017). Estudio comparativo del uso de tecnologías de información y comunicación en pymes y grandes empresas. Revista RAITES (antes Panorama Administrativo) Vol. 3 No. 7. Julio-diciembre 2017

Riascos Erazo, S.C. (2016). Inversión en Tecnologías de la Información y las Comunicaciones y su relación con en el direccionamiento estratégico de las PYMES de Santiago de Cali-Colombia. Revista lbérica de Sistemas y Tecnologías de Información. RISTI, N. ${ }^{o}$ 18, 06/2016. DOI: 10.17013/risti.18.1-17

Rodríguez Melo, GE. (2012). Apropiación y uso de las Tecnologías de la Información y las Comunicaciones (TIC) en la Micro, Pequeña y Mediana Empresa (MiPyME) de Latinoamérica: Análisis de Experiencias regionales. En: https://www.researchgate.net/publication/294732627 
Sánchez Limón, M.L y De la Garza Cárdenas, M.H. (2018). Tecnologías de información y desempeño organizacional de las pymes del noreste de México. Revista Venezolana de Gerencia $(R V G)$ Año. 23, No. 82, 2018, pp 298 - 313. Maracaibo, Venezuela. ISSN 1315-9984. En: https://www.researchgate.net/publication/327068741

Sariannidis, N., Lemonakis, C., Garefakalis, A., Paraskevas, T. (2019). Manufacturing SMEs competitiveness against the crisis: Management characteristics and new perspectives. Int. $J$ Sup. Chain Mgt 8(4). 2019.

Troncoso, I., López, I., Fischer, C., Luzio, Á., Muñoz, V., \& Carrasco, M. (2013). Estudio descriptivo de gestión organizacional usada en 30 clínicas veterinarias de la octava región de Chile. 